\title{
Counterfactual distribution of cat states
}

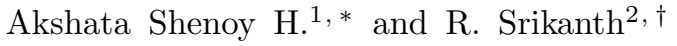 \\ ${ }^{1}$ Electrical Communication Engg. Dept., IISc, Bengaluru, India \\ ${ }^{2}$ Poornaprajna Institute of Scientific Research, Bengaluru, India
}

\begin{abstract}
In the counterfactual cryptography scheme proposed by Noh (2009), the sender Alice probabilistically transmits classical information to the receiver Bob without the physical travel of a particle. Here we generalize this idea to the distribution of quantum entanglement. The key insight is to replace their classical input choices with quantum superpositions. We further show that the scheme can be generalized to counterfactually distribute multi-partite cat states.
\end{abstract}

PACS numbers: 03.67.Hk,03.65.Ud

\section{INTRODUCTION}

Counterfactual quantum communication [1, 2] allows information to be transmitted even without the physical travel of a particle. It is based on interaction-free measurements, where one tries to detect a quantum object without directly interrogating it 3 . Ref. 4 proposed a method to increase the efficiency (of 50\%) of the counterfactual effect in the original Elitzur-Vaidman protocol [3] towards 1 by concatenating unbalanced beam splitters, each of which effects the transformation $|0\rangle \rightarrow$ $\cos \theta|0\rangle+\sin \theta|1\rangle$ and $|1\rangle \rightarrow-\sin \theta|0\rangle+\cos \theta|1\rangle$. This constitutes the quantum Zeno effect (QZE), wherein repeated measurements or 'interrogation' of an initial state freeze evolution.

Briefly, this works as follows: light in state $|0\rangle$ is incident on a beam splitter. An obstacle is placed in the output arm $|1\rangle$. The obstacle in the state "block" eliminates the corresponding amplitude in the superposition, whereas in the state "pass", it does nothing. Chaining the above interferometric action leads to the evolution:

$$
\begin{aligned}
\mid \text { block }\rangle|0\rangle & \left.\longrightarrow \cos ^{L} \theta \mid \text { block }\right\rangle|0\rangle \\
\mid \text { pass }\rangle|0\rangle & \longrightarrow \text { pass }\rangle(\cos (L \theta)|0\rangle+\sin (L \theta)|1\rangle),
\end{aligned}
$$

where $L$ is the number of interferometric cycles, and the unnormalized state in the first equation indicates absorption at the obstacle. Setting $\theta=\frac{\pi}{2 L}$, let $L \rightarrow \infty$. The result has full counterfactual efficiency in that the obstacle set in block mode always produces a distant detection in the output $|0\rangle$ arm.

Counterfactuality has also been applied to both cryptography [5] and computation [6, 7]. Since the proposal of the counterfactual key distribution protocol Noh-2009 (N09) 8, there has been an upsurge of interest in this area, leading to contributions devoted to improving its efficiency [9, 10], to security considerations under various attacks [11 13, to modified communication schemes [14, 15], applications [16-18 and experimental proposals or realizations 19 .

\footnotetext{
* akshata@ece.iisc.ernet.in

$\dagger$ srik@poornaprajna.org
}

\section{COUNTERFACTUAL QUANTUM STATE TRANSFER}

The method of Eq. (1) can be used for direct and deterministic communication of classical bits from Bob located at the obstacle to Alice located at the interferometer output arms. But as a communication protocol, this method is not counterfactual for the case "pass" since the photon encounters no obstacle. To make both bit values counterfactual, 14 proposes the chained quantum Zeno effect (CQZE) set-up, which nests the above $L$-chained interferometer within a chain of $M$ "outer" unbalanced beam splitters, in such a way that at each inner cycle, the outcome arm corresponding to "pass" would lead to detection at a detector $\Delta$. One sequentially evolves the state through each of the outer interferometric cycles, which gives a recursion relation for amplitudes for each cycle, analogous to Eq. (2). By suitable choice of $L$ and $M$, one can obtain direct communication of bits from Alice to Bob. This is argued to be fully counterfactual in the sense that Bob's both "pass" and "block" choices correspond to blocking actions. (Regarding this interpretation, see Refs. [20].)

Now, the method of Eq. (1) can be readily adapted to one for direct communication of qubits (rather than bits) from Bob to Alice by letting the obstacle to be in the superposition state $\alpha \mid$ pass $\rangle+\beta \mid$ block $\rangle$ [21, 22. An initial state $(\alpha \mid$ pass $\rangle+\beta \mid$ block $\rangle) \otimes|0\rangle$ of the obstacle and the particle evolves to $(\alpha \mid$ pass $\rangle+\beta \mid$ block $\rangle) \otimes$ $(\cos \theta|0\rangle+\sin \theta|1\rangle)$ after the particle passes through the first beam splitter. Following the particle's interaction with the obstacle placed in arm $|1\rangle$ after the beam splitter, the state becomes $\sqrt{1-\beta^{2} \sin ^{2}(\theta)}[\alpha|\operatorname{pass}\rangle(\cos \theta|0\rangle+$ $\sin \theta|1\rangle)+\beta \mid$ block $\rangle|0\rangle]$. Here the global pre-factor, which is the square root of the particle's survival probability, comes from the fact that $\beta^{2} \sin ^{2}(\theta)$ is the probability that the photon is absorbed at the obstacle. Just after the second beam splitter, but before encountering the obstacle, the state is $\sqrt{1-\beta^{2} \sin ^{2}(\theta)}[\alpha|\operatorname{pass}\rangle(\cos (2 \theta)|0\rangle+$ $\sin (2 \theta)|1\rangle)+\beta \mid$ block $\rangle(\cos \theta|0\rangle+\sin \theta|1\rangle)]$. Proceeding thus, just after the application of $L$ beam splitter pas- 
sages, the state transforms as:

$$
\begin{aligned}
& (\alpha \mid \text { pass }\rangle+\beta \mid \text { block }\rangle)|0\rangle \rightarrow\left[1-\beta^{2} \sin ^{2}(\theta)\right]^{(L-1) / 2} \times \\
& \quad[\alpha \mid \text { pass }\rangle(\cos (L \theta)|0\rangle+\sin (L \theta)|1\rangle) \\
& \quad+\beta \mid \text { block }\rangle(\cos \theta|0\rangle+\sin \theta|1\rangle)] \\
& \quad \rightarrow \alpha \mid \text { pass }\rangle|1\rangle+\beta \mid \text { block }\rangle|0\rangle
\end{aligned}
$$

where the right-arrow in the last equation indicates taking the limit $L \rightarrow \infty$ with $\theta=\frac{\pi}{2 L}$. (Note that this limit restores the normalization.)

If the $L$-cycle is nested in an $M$-cycle as described above, then analogous to the superposition (2), the final state after passing through the $M$ outer cycles, by adjusting $L$ and $M$, can be brought to the form:

$$
\left.\left.\left|\Psi_{\text {fin }}\right\rangle \approx \alpha \mid \text { pass }\right\rangle|x\rangle-\beta \mid \text { block }\right\rangle|y\rangle,
$$

where $x, y$ label the output arms of the $M$ th outer interferometer. Note that the entangled state $\left|\Psi_{\text {fin }}\right\rangle$ depends on Bob's initial state $\alpha \mid$ block $\rangle+\beta \mid$ pass $\rangle$. With a Hadamard and 1-bit classical communication, Alice can recover Bob's state in the $\{|x\rangle,|y\rangle\}$ basis (see later below). This forms the essence of the protocol of Guo et al. 22] for quantum state transfer. It requires only 1 bit for deterministic quantum state transfer, unlike standard quantum teleportation, which requires 2 bits, because teleportation uses a standard Bell state as the entanglement resource, whereas the above protocol uses the statedependent entanglement (3). We note that even without the classical communication, the protocol succeeds half the time, such that the fidelity of the transmitted mixed state is 1 for polar qubits, falling gradually as $|\cos (\theta)|$ towards 0 for equatorial $\left(\theta=\frac{\pi}{2}\right)$ qubits of the Bloch sphere in the $\{\mid$ block $\rangle, \mid$ pass $\rangle\}$ basis.

That the counterfactual quantum protocol to transmit a classical bit [14] is direct and deterministic suggests that a protocol for quantum state transfer built on top of it can also be that way. Ref. 21] proposes one way to do this: a polarization-based "dual" CQZE scheme, which essentially first entangles Alice's and Bob's input during a "counterfactual CNOT operation", and then disentangles them using a second counterfactual CNOT in such a way that the combined action swaps Alice's state $|0\rangle$ with Bob's state $\alpha \mid$ pass $\rangle+\beta \mid$ block $\rangle$ deterministically. Note that the swap of two generic qubit states requires three CNOTs, in constrast to the two CNOTs that suffice for the states considered here.

In the present work, we propose to use N09 instead of the CQZE system, as the basis for counterfactual distribution of entanglement. Like the state (3), this entanglement will depend on the initial superposition states of Bob's obstacle and Alice's input. Unlike them, however, in our case the state-dependent entanglement is generated probabilistically. Once generated, this entanglement can be used for deterministic quantum state transfer with a 1-bit communication, as in the Guo et al. protocol. Though our method has a lower yield than the methods of Refs. [21, 22, still our experimental set-up is sim- pler in that we do not require a large chaining of beamsplitters. Further, the schemes [21, 22] pertain to the bipartite case, whereas our method is generalized to one that can counterfactually distribute multipartite quantum states, in particular $(N+1)$-partite cat states, where $N$, an integer greater than 1 , is the number of players who initiate the protocol.

The remaining article is structured as follows. After briefly describing the N09 protocol in Section III, we show in Section IV how to modify it for the purpose of bipartite entanglement distribution, essentially by replacing bits $a$ and $b$ by qubits, and correspondingly replacing $R_{a}$ and $R_{b}$, the classical random number generators, by their quantum counterparts, which can generate superposition states. Section $[\mathrm{V}]$ discusses using the counterfactually distributed entanglement for the purpose of quantum state transfer, comparing our method with those of counterfactual schemes of [21, 22] and standard quantum teleportation. In Section VI we extend the bipartite scheme to that of generating multipartite cat states, by introducing a quantum network with a star topology. In Section VII. we discuss candidate physical systems for practical implementation. Finally we discuss some implications of our scheme for physical interpretation, the extension of the scheme of Ref. 22] for the counterfactual distribution of cat states, and offer some conclusions in Section VIII.

\section{COUNTERFACTUAL COMMUNICATION OF CLASSICAL BITS: THE N09 PROTOCOL}

To set the background, we briefly describe N09 as follows. The basic set-up of the N09 is similar to the one shown in Fig. 1. The sender (Alice) and the receiver (Bob) are connected to each other through $\operatorname{arm} B$ of a Michelson interferometer. The $\operatorname{arm} A$ is internal to $\mathrm{Al}-$ ice's station and a photon travelling along this path is reflected using a Faraday mirror (FM). Alice's station also consists of a single photon source $(S)$ which prepares polarization states in the vertical $(V)$ or horizontal $(H)$ direction, based on the output $a$ of a random number generator $R_{a}$. Bob's module $Q$ consists of detector $D_{B}$ and an FM which absorbs or reflects photons in a polarization-dependent way based on the state of a switch. The switch state is $P$ ("pass $V$ and block $H$ ") or $B$ ("block $V$ and pass $H$ "), as determined by the output $b$ of a random number generator $R_{b}$. The protocol runs as follows:

1. Depending on the random bit $a$, Alice prepares single photon states randomly in $V$ or $H$ polarization, and transmits them to Bob, who applies $P$ or $B$ actions randomly based on the random bit $b$.

2. The possibilities of Alice's and Bob's random switching actions, and the corresponding condi- 
tional probabilities, are:

\begin{tabular}{c||c|c|c}
\hline (Alice, Bob) & $D_{1}$ & $D_{2}$ & $D_{B}$ \\
\hline$(V, P)$ or $(H, B)$ & 0 & 1 & 0 \\
\hline$(V, B)$ or $(H, P)$ & $R T$ & $R^{2}$ & $T$ \\
\hline
\end{tabular}

In the first case, deterministic detection of $D_{2}$ because of constructive interference between the two reflected photons. Here $R$ and $T$ are the coefficients of reflectance and transmittance respectively of the beamsplitter (BS), and satisfy $R+T=1$.

3. After the protocol run, Alice announces instances where she made $D_{1}$ detections, and use the polarization $(V$ or $H)$ as a secret bit. The $D_{2}$ detections are used for security check.

The case of $D_{1}$ is counterfactual in the sense that the particle did not physically travel to Bob's station, and his blocking action leads to a remote detection by Alice. The counterfactual yield is $R T / 2$ and, in the noiseless case, would represent the positive key rate.

\section{COUNTERFACTUAL BIPARTITE ENTANGLEMENT GENERATION: A NEW SCHEME}

We shall now adapt N09 to distribute quantum entanglement. We begin with the demonstration of our idea to counterfactually generate bi-partite entanglement. The basic idea can be indicated again using Figure 1 . The key new element is that $R_{a}$ and $R_{b}$ are now quantum random number generators that produce qubits $a$ and $b$. Let qubit $b$ be given by $|\psi\rangle_{b}=\alpha|P\rangle_{b}+\beta|B\rangle_{b}$ and $a$ by $|\phi\rangle_{a}=\mu|V\rangle_{a}+\nu|H\rangle_{a}$. Then, the initial joint states of the $R_{a}, R_{b}$, Bob's detector and the optical field can be written as,

$$
\begin{aligned}
\left|\Psi_{0}\right\rangle & =|\phi\rangle_{a}|0\rangle_{D_{B}}|\psi\rangle_{b}|\Psi\rangle_{A B} \\
& =\left(\mu|V\rangle_{a}+\nu|H\rangle_{a}\right)|0\rangle_{D_{B}}\left(\alpha|P\rangle_{b}+\beta|B\rangle_{b}\right)|0\rangle_{A}|0\rangle_{B}
\end{aligned}
$$

where $\left|\Psi_{0}\right\rangle_{A B} \equiv|0\rangle_{A}|0\rangle_{B}$ is the initial vacuum state of the channel, represented by the interferometer arms.

Alice's random number generator $R_{a}$ now determines the polarization state of the photon. Under interaction with $R_{a}$ and BS, the state $\left|\Psi_{0}\right\rangle$ evolves to:

$$
\begin{aligned}
\left|\Psi_{1}\right\rangle= & \left(\alpha|P\rangle_{b}+\beta|B\rangle_{b}\right)|0\rangle_{D_{B}} \otimes \\
& \left(\mu|V\rangle_{a}\left[i \sqrt{R}|V\rangle_{A}|0\rangle_{B}+\sqrt{T}|0\rangle_{A}|V\rangle_{B}\right]\right. \\
+ & \left.\nu|H\rangle_{a}\left[i \sqrt{R}|H\rangle_{A}|0\rangle_{B}+\sqrt{T}|0\rangle_{A}|H\rangle_{B}\right]\right)
\end{aligned}
$$

Let $|0\rangle_{D_{B}}$ and $|Y\rangle_{D_{B}}$ represent the initial state of Bob's detector, and the state of his detector after detecting a photon. We represent the switch action on Bob's side

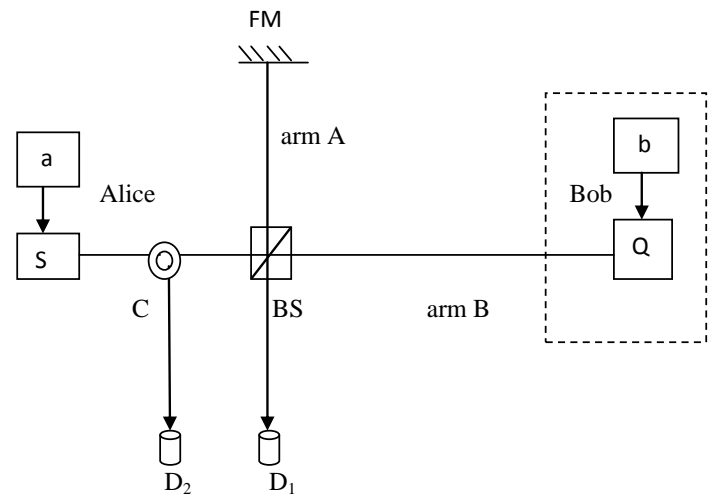

FIG. 1. Basic schematic for N09, where $a$ and $b$ take random classical bit values. For its adaptation to counterfactual generation of bipartite entanglement, $a$ and $b$ are qubits that can exist in arbitrary superposition states. $C$ denotes a circulator. The switch $Q$ implements pass- $V$-block- $H$ or pass- $H$-block- $V$ on the incoming photon, depending on $b$. To distribute entanglement counterfactually, $Q$ is entangled with Bob's input $b$.

thus:

$$
\begin{aligned}
|P\rangle_{b}|V\rangle_{B}|0\rangle_{D_{B}} & \rightarrow|P\rangle_{b}|V\rangle_{B}|0\rangle_{D_{B}} \\
|P\rangle_{b}|H\rangle_{B}|0\rangle_{D_{B}} & \rightarrow|P\rangle_{b}|0\rangle_{B}|Y\rangle_{D_{B}} \\
|B\rangle_{b}|V\rangle_{B}|0\rangle_{D_{B}} & \rightarrow|B\rangle_{b}|0\rangle_{B}|Y\rangle_{D_{B}} \\
|B\rangle_{b}|H\rangle_{B}|0\rangle_{D_{B}} & \rightarrow|B\rangle_{b}|H\rangle_{B}|0\rangle_{D_{B}}
\end{aligned}
$$

Post-selected on Bob's detector being found in the state $|0\rangle_{D_{B}}$, the optical field in state $\left|\Psi_{1}\right\rangle$ evolves under (7) to the un-normalized state

$$
\begin{aligned}
\left|\Psi_{2}\right\rangle= & \alpha|P\rangle_{b}\left(\mu|V\rangle_{a}\left[i \sqrt{R}|V\rangle_{A}|0\rangle_{B}+\sqrt{T}|0\rangle_{A}|V\rangle_{B}\right]\right. \\
& \left.+\nu|H\rangle_{a}\left[i \sqrt{R}|H\rangle_{A}|0\rangle_{B}\right]\right) \\
+ & \beta|B\rangle_{b}\left(\mu|V\rangle_{a}\left[i \sqrt{R}|V\rangle_{A}|0\rangle_{B}\right]\right. \\
& \left.+\nu|H\rangle_{a}\left[i \sqrt{R}|H\rangle_{A}|0\rangle_{B}+\sqrt{T}|0\rangle_{A}|H\rangle_{B}\right]\right),
\end{aligned}
$$

with probability $P\left(D_{B}\right)$ for Bob's detector being found in state $|Y\rangle_{D_{B}}$ being

$$
P\left(D_{B}\right)=1-\left|\left\langle\Psi_{2} \mid \Psi_{2}\right\rangle\right|^{2}=\left(|\alpha \nu|^{2}+|\beta \mu|^{2}\right) T .
$$

In the last phase, the light re-enters Alice's system. It is convenient to represent the $\mathrm{BS}$ transformation in the return path, together with the reflection on Bob's side, by:

$$
\begin{aligned}
& |0\rangle_{A}|x\rangle_{B} \longrightarrow \sqrt{R}\left|D_{1}^{x}\right\rangle+i \sqrt{T}\left|D_{2}^{x}\right\rangle \\
& |x\rangle_{A}|0\rangle_{B} \longrightarrow i \sqrt{T}\left|D_{1}^{x}\right\rangle+\sqrt{R}\left|D_{2}^{x}\right\rangle,
\end{aligned}
$$

where $x=V, H$, and $\left|D_{j}^{x}\right\rangle$ denotes the state of detector $D_{j}$ when it detects a photon of polarization $x$.

Thereby the post-selected state $\left|\Psi_{2}\right\rangle$ evolves to $\left|\Psi_{3}\right\rangle \equiv$ $\left|\Psi_{3}^{\prime}\right\rangle|0\rangle_{D_{B}}$, where 


$$
\begin{aligned}
\left|\Psi_{3}^{\prime}\right\rangle & =\alpha|P\rangle_{b}\left(\mu|V\rangle_{a}\left|D_{2}^{V}\right\rangle+\nu|H\rangle_{a}\left[i \sqrt{R}\left(i \sqrt{T}\left|D_{1}^{H}\right\rangle+\sqrt{R}\left|D_{2}^{H}\right\rangle\right)\right]\right) \\
& +\beta|B\rangle_{b}\left(\mu|V\rangle_{a}\left[i \sqrt{R}\left(i \sqrt{T}\left|D_{1}^{V}\right\rangle+\sqrt{R}\left|D_{2}^{V}\right\rangle\right)\right]+\nu|H\rangle_{a}\left|D_{2}^{H}\right\rangle\right) .
\end{aligned}
$$

If the superpositions in $a$ and $b$ are replaced by their projective measurements prior to the photon entering the interferometer, then the present protocol reduces to N09, in particular reproducing the conditional probabilities (4). For example, from Eq. (9), we have $P\left(D_{B}\right)=$ $\left(|\alpha \nu|^{2}+|\beta \mu|^{2}\right) T$. From Bayesian analysis we have $P\left(D_{B}\right)=P\left(D_{B} \mid H_{a} P_{b}\right) P\left(H_{a} P_{b}\right)+P\left(D_{B} \mid V_{a} B_{b}\right) P\left(V_{a} B_{b}\right)$. Now $P\left(H_{a} P_{b}\right)=P\left(H_{a}\right) P\left(P_{b}\right)=|\alpha \nu|^{2}$ and $P\left(V_{a} B_{b}\right)=$ $P\left(V_{a}\right) P\left(B_{b}\right)=|\beta \mu|^{2}$, where $P\left(H_{a}\right)$ etc., are probabilities to obtain the designated outcome under projective measurement of the corresponding device $\left(R_{a}\right.$ or $\left.R_{b}\right)$. Equating these two expressions for $P\left(D_{B}\right)$ and noting that this holds true for arbitrary protocol parameters, we find that $P\left(D_{B} \mid H_{a} P_{B}\right)=P\left(D_{B} \mid V_{a} B_{b}\right)=T$, as given in Eq. (4).

Now, conditioned on $D_{1}$ being detected (which corresponds to the projector $\sum_{H, V}\left|D_{1}^{x}\right\rangle\left\langle D_{1}^{x}\right|$ ), we have the counterfactual situation with the resulting (unnormalized) state being

$$
|\xi\rangle=\sqrt{R T}\left(\alpha \nu|H\rangle_{a}|P\rangle_{b}+\beta \mu|V\rangle_{a}|B\rangle_{b}\right),
$$

where the counterfactual yield is seen to be

$$
P\left(D_{1}\right)=R T\left(|\alpha \nu|^{2}+|\beta \mu|^{2}\right) .
$$

We note that the beam splitter function $\sqrt{R T}=$ $\sqrt{R(1-R)}$ does not determine the degree of entanglement generated counterfactually, which depends only on the initial superposition states held by Alice and Bob. A $D_{B}$ detection results in the entangled (unnormalized) state $T\left(\alpha \nu|H\rangle_{a}|P\rangle_{b}+\beta \mu|V\rangle_{a}|B\rangle_{b}\right)$, which differs from state (12) by a constant factor. However it is not counterfactual. Similarly, a $D_{2}$ detection, which happens with probability

$$
P\left(D_{2}\right)=|\alpha|^{2}\left(|\mu|^{2}+|\nu|^{2} R^{2}\right)+|\beta|^{2}\left(|\nu|^{2}+|\mu|^{2} R^{2}\right),
$$

non-counterfactually produces the state $\alpha\left(\mu|V\rangle_{a}+\right.$ $\left.\nu R|H\rangle_{a}\right)|P\rangle_{b}+\beta\left(i \mu R|V\rangle_{a}+\nu|H\rangle_{a}\right)|B\rangle_{b}$, which is in general entangled.

Intuitively, the above scheme can be thought of as tripartite entanglement being established between the photon, Alice's device $R_{a}$ and Bob's device $R_{b}$, after which the photon is projected out in a suitable basis, leaving $R_{a}$ and $R_{b}$ entangled.

\section{COUNTERFACTUAL QUANTUM INFORMATION TRANSFER IN THE NEW SCHEME}

By means of the state-dependent entanglement so obtained, a quantum state transfer can be implemented either from Alice to Bob or from Bob to Alice, with a 1-bit communication. The sender puts the input in the required unknown state, while the receiver prepares her/his input in an equal-weighted superposition in the default basis. For example, suppose that it is desired to transfer Alice's state $|\phi\rangle_{a}=\mu|V\rangle_{a}+\nu|H\rangle_{a}$ to Bob. Bob prepares his input state with $\alpha=\beta=\frac{1}{\sqrt{2}}$. From Eq. $\sqrt{12}$, we see that Alice and Bob (probabilistically) will share the state $\nu|H\rangle_{a}|P\rangle_{b}+\mu|V\rangle_{a}|B\rangle_{b}$. Applying a Hadamard on Alice's qubit in the $\{V, H\}$ basis results in the state

$$
\frac{|V\rangle_{a}}{\sqrt{2}}\left(\nu|P\rangle_{b}-\mu|B\rangle_{b}\right)+\frac{|H\rangle_{a}}{\sqrt{2}}\left(\nu|P\rangle_{b}+\mu|B\rangle_{b}\right) .
$$

Measuring in the $\{V, H\}$ basis, and depending on whether Alice finds outcome $V$ or $H$, she communicates a 1-bit message to Bob, which instructs him to apply the Pauli operation $Z$ or $I$ in the $\{P, B\}$ basis. Bob is then left with the reconstructed state: $|\phi\rangle_{b} \equiv \mu|B\rangle_{b}+\nu|P\rangle_{b}$.

This situation is analogous to that encountered in Ref. [22], except that there the state-dependent entanglement is deterministically generated (in the asymptotic limit of the number of beam splitters), whereas in our case, it is generated probabilistically, with probability $P\left(D_{1}\right) \equiv \frac{R T}{2}=\frac{R(1-R)}{2}$, as follows from Eq. 13). Denoting by the term $C_{q}$ the number of photons consumed (or runs required) on average per counterfactual event, we have $C_{q}=\frac{1}{P\left(D_{1}\right)}=\frac{2}{R(1-R)}$. The minimum value of $C_{q}$ is $C_{q}^{\min } \equiv 8$ photons (or runs), which is attained at $R=\frac{1}{2}$. In other words, a balanced beam splitter minimizes the quantum resource used. The situation is slightly different for the average classical communication required, as shown below.

Assuming the communication channel to be noiseless, and the light to consist of single-photons, the average communication cost for generating an entangled pair is:

$$
C=h\left(\frac{R(1-R)}{1+R}\right) \frac{1+R}{R(1-R)},
$$

where $h(x) \equiv-x \log _{2}(x)-(1-x) \log _{2}(1-x)$ is the Shannon binary entropy. From Eq. (15), we see that cost $C$ increases without bound as $R \rightarrow 0$ or $R \rightarrow 1$. This can be anticipated, referring to Eq. (4), where we find that the number of counterfactual $\left(D_{1}\right)$ events tend to vanish in either limit, thereby necessitating a large communication cost. Note that since each run requires at most one bit communication, therefore as a thumb rule, $\operatorname{cost} C$ in 15) cannot be larger than $C_{q}$, at a given $R$.

To derive (15), suppose that $n$ runs have been conducted, and Bob's input state is chosen with $\alpha=\beta=\frac{1}{\sqrt{2}}$ in each run. Then the probabilities for the different outcomes $D_{1}, D_{2}$ and $D_{B}$ are just the conditional probabilities in Eq. (4) with a factor $\frac{1}{2}$, independent of $\mu$ and $\nu$, as 
follows from Eqs. (9), 13) and 14). For sufficiently large $n$, about $\frac{n T}{2}$ detection events $D_{B}$ occur, during which Alice has no detection, but obviously does not need to communicate this information to Bob. Thus on average, only on the remaining $n_{B} \equiv n P\left(\neg D_{B}\right) \equiv n\left(1-\frac{T}{2}\right)=\frac{n(1+R)}{2}$ of events does Alice need to communicate to Bob about whether a $D_{1}$ or $D_{2}$ event happened.

Detections $D_{1}$ and $D_{2}$ occur among these events, with probability $P\left(D_{1}\right) \equiv R T / 2=\frac{R(1-R)}{2}$ and $P\left(D_{2}\right) \equiv$ $\frac{1+R^{2}}{2}$, respectively, as seen from Eqs. 13 and 14 . Therefore the probability of a $D_{1}$ or $D_{2}$ event in the outcome space of non- $D_{B}$ events is $p^{\prime}\left(D_{1}\right) \equiv$ $P\left(D_{1}\right) / P\left(\neg D_{B}\right)=\frac{R(1-R)}{1+R}$ and $p^{\prime}\left(D_{2}\right)=1-p^{\prime}\left(D_{1}\right)=$ $\frac{1+R^{2}}{1+R}$, respectively. Therefore, the bit string about $n_{B}$ long, corresponding to events when Alice needs to communicate, can be compressed (by Shannon's noiseless channel coding theorem [23]) to $n_{B}^{*} \equiv h\left[p^{\prime}\left(D_{1}\right)\right] n_{B}$ bits. On average, these events resulted in the counterfactual distribution of $n_{1} \equiv n P\left(D_{1}\right)=\frac{n}{2} R(1-R)$ statedependent entangled states. The ratio $n_{B}^{*} / n_{1}$ gives the required expression for the average classical communication cost (15).

To minimize cost $C$, note that the rhs in Eq. (15) has the form $h(\xi) / \xi$, which falls monotonically as $\xi$ goes from 0 to 1 . Now the argument of $h(\cdot)$ in Eq. (15), namely $\xi \equiv R(1-R) /(1+R)$, takes values only in the range $[0,1]$. Thus the required minimum for $C$ is obtained by minimizing $\xi$ as a function of $R$, which is seen to occur at $R=\sqrt{2}-1 \approx 0.414$, with the corresponding average communication cost being $\left.C^{\min } \equiv C\right|_{R=\sqrt{2}-1} \approx 3.85$ bits. We note that $C^{\text {min }} \leq C_{q}^{\text {min }}$, and that the latter minimum occurs at $R=\frac{1}{2}$. For the case of a balanced beam splitter, we find $\left.C\right|_{R=0.5} \approx 3.9>C^{\text {min }}$. The minimum number of bits of classical communication required on average using our counterfactual teleportation is $C^{\min }+1 \approx 4.85$ bits. This is larger than the 2 bits sufficient (and necessary) for standard quantum teleportation, 1 bit for the 22] protocol and 0 bit for the protocol of [21]. Of course, these three schemes being deterministic, $C_{q}=1$ for each of them.

\section{EXTENSION TO CAT STATES}

We now show how to extend the above method of counterfactual generation of bipartite entanglement to $(N+1)$-partite entanglement. It will be convenient here to regard the 'quantized' version of (4) as the action of a propagator. Then the 'partial propagator' relevant here has the action:

$$
\begin{aligned}
|P\rangle_{b}|H\rangle_{a}|\Psi\rangle_{A B} & \rightarrow \sqrt{R T}|P\rangle_{b}|H\rangle_{a}\left|D_{1}^{H}\right\rangle \\
|B\rangle_{b}|V\rangle_{a}|\Psi\rangle_{A B} & \rightarrow \sqrt{R T}|B\rangle_{b}|V\rangle_{a}\left|D_{1}^{V}\right\rangle
\end{aligned}
$$

where by 'partial propagator' we mean that the evolved final state is post-selected only on $D_{1}$ detections.

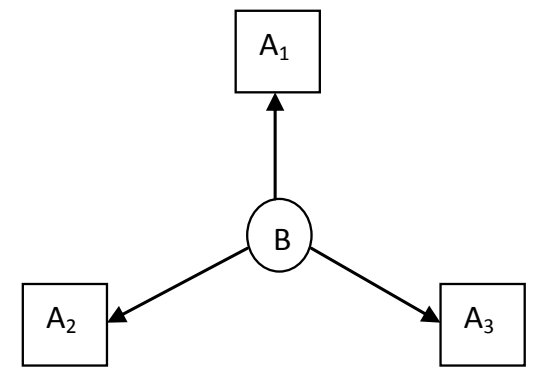

FIG. 2. Multipartite counterfactual entanglement distribution: We consider a star topology, with a single Bob at the network hub, who shares the set-up of Figure 1 with each of $N$ (here: $N=3$ ) Alices. Bob's quantum choice must be jointly applied to all $N$ channels on his side.

Now we can generalize the set-up of Figure 1 to counterfactually distribute an $(N+1)$-cat state, as shown in Figure 2, which illustrates the network for the distribution among four parties. Typically, it is described by a star topology, with Bob at the network hub and the remaining players (who we call Alice ${ }_{1}$, Alice 2 , et al., for convenience) situated at the spoke nodes. Bob is connected according to the circuit of Figure 1 with each Alice $_{j}$ separately. The only requirement is that Bob's quantum device $R_{b}$ makes a common choice for all Alices' particles.

For instance, considering the case of $N=2$, the initial state of the two Alices and Bob, given by $\left|\Psi_{4}\right\rangle=$ $\bigotimes_{j=1}^{2}\left(\mu_{j}|V\rangle+\nu_{j}|H\rangle\right)_{a_{j}} \otimes\left(\alpha|P\rangle_{b}+\beta|B\rangle_{b}\right)|\Psi\rangle_{A_{1} B_{1}}|\Psi\rangle_{A_{2} B_{2}}$, where $|\psi\rangle_{A_{1} B_{1}}\left(|\psi\rangle_{A_{2} B_{2}}\right)$ indicate the optical channel between Alice ${ }_{1}$ (Alice 2 ) and Bob, evolves under the partial propagator (16) to

$$
\left|\Psi_{\text {cat }}\right\rangle_{a_{1} a_{2} b}=R T\left(\mu_{1} \mu_{2} \beta|V V B\rangle+\nu_{1} \nu_{2} \alpha|H H P\rangle\right)_{a_{1} a_{2} b},
$$

with the counterfactual yield being $(R T)^{2}\left(\left|\mu_{1} \mu_{2} \beta\right|^{2}+\right.$ $\left.\left|\nu_{1} \nu_{2} \alpha\right|^{2}\right)$.

It is straightforward to extend the above exercise to $N$ Alice's, thereby producing a $(N+1)$-cat state between these Alices and Bob. The initial state is taken to be

$$
\bigotimes_{j=1}^{N}\left(\mu_{j}|V\rangle_{j}+\nu_{j}|H\rangle_{j}\right)_{a_{j}} \otimes(\alpha|P\rangle+\beta|B\rangle)_{b},
$$

which evolves under the evolution (16), corresponding to post-selection on all Alices detecting $D_{1}$, to the state:

$$
\left|\Psi_{7}\right\rangle=(R T)^{N / 2} \alpha \Pi_{\mu}|V\rangle_{a}^{\otimes N}|B\rangle_{b}+\beta \Pi_{\nu}|H\rangle_{a}^{\otimes N}|P\rangle_{b},
$$

where $\Pi_{\mu} \equiv \mu_{1} \mu_{2} \cdots \mu_{N}$ and $\Pi_{\nu} \equiv \nu_{1} \nu_{2} \cdots \nu_{N}$.

By setting $\alpha=\beta=\frac{1}{\sqrt{2}}$ and $\mu_{j}=\nu_{j}=\frac{1}{\sqrt{2}}$, we obtain a maximally entangled cat state, with yield $\left(\frac{R T}{2}\right)^{N}$, indicating an exponential fall-off. 


\section{EXPERIMENTAL IMPLEMENTATION}

Recent experimental advances in quantum information processing and in the demonstration of quantum coherence in mesoscopic systems has been impressive, examples being trapped ion systems 24 and large biomolecules 25. The key experimental challenge to implement our proposal is putting Bob's obstacle in the superposition of "pass $\mathrm{H}$, block V" and "pass V, block $\mathrm{H}$ ", which is a highly non-trivial requirement.

Considerable experimental simplification happens in our scheme, while at the same time preserving essentially the same idea for counterfactual distribution of entanglement, if we employ the semi-counterfactual quantum key distribution (ScQKD) protocol [15, rather than N09, as the point of departure for counterfactual communication from classical to quantum information. Moreover, a Mach-Zehnder interferometer version, rather than that using the Michelson interferometer, may be advantageous for an experimental demonstration, as is done in the experimental realization of N09 [19].

For the present purpose, the ScQKD scheme is similar to N09 except that secret bits are encoded in terms of plain "pass" and "block" actions, rather than the polarization-specific blockade actions. The Michelson interferometer in Figure 1 remains the same, except that the fixed mirror at the top is replaced by a polarizationindependent pass/block set-up similar to Bob's. This set-up now forms Alice's module. Counterfactual bits are generated in $D_{1}$ detections and happen only when precisely one of Alice and Bob applies "pass" and the other "block". To be precise, the bits are counterfactual only with respect to the person applying the blockade. Thus it is counterfactual only on part of the runs with respect to the distant agent, Bob, hence the name semicounterfactual. By replacing Alice's and Bob's classical choices by superpositions of the type $\alpha_{j} \mid$ pass $\rangle+\beta_{j} \mid$ block $\rangle$ ( $j=A, B)$, one counterfactually and probabilistically generates entanglement of the (unnormalized) form

$$
\left.\left.\left.\left.\alpha_{A} \beta_{B} \mid \text { pass }\right\rangle_{A} \mid \text { block }\right\rangle_{B}+\beta_{A} \alpha_{B} \mid \text { block }\right\rangle_{A} \mid \text { pass }\right\rangle_{B},
$$

where the first and second registers refer to Alice's and Bob's systems.

We note that in ScQKD, both bits are counterfactual with respect to one of Alice and Bob. Moreover, Alice and Bob are spatially separated. Both these features are relaxed in the Kwiat et al. scheme [26] (where only the "block" option is counterfactual with respect to Alice or Bob), though at the price of requiring a chain of beam splitter actions. Thus, an alternative, and in some ways, even simpler first step towards experimental implementation of counterfactual distribution of entanglement would be the semi-counterfactual generation of entanglement of the type 2.

We suggest two broad ways to experimentally achieve such superposition states 20 of the obstacle: one using spatial Schrödinger cat states and the other using a special kind of electromagnetically induced transparency (EIT) systems.

In the first way, Bob's system is put in the superposition of being present and absent in the communication channel. The presence of the obstacle would correspond to the "block" action and the absence to the "pass" action on the photon. Trapped ions 24] are possible candidate systems here.

The second way to realize the quantum obstacle is as a mesoscopic system placed in a quantum superposition of being transparent and opaque to photons passing through the channel. A possible candidate here is of a cloud of Rydberg atom confined in a trapping potential localized within the blockade radius 27. Owing to the dipole-dipole interaction between the atoms, they show collective excitation of the form $|R\rangle=\frac{1}{\sqrt{N}} \sum_{j=1}^{N}\left|g_{1}, g_{2}, \cdots, r_{j}, \cdots g_{N}\right\rangle$, where $\left|g_{k}\right\rangle$ and $\left|r_{k}\right\rangle$ are the ground and Rydberg excited states of the $k$ th atom. Otherwise they remain in the ground state $|G\rangle \equiv\left|g_{1}, g_{2}, \cdots, g_{N}\right\rangle$. The transition between $\left|g_{k}\right\rangle$ and $\left|r_{k}\right\rangle$ is tuned to the energy of the photon used for communication.

In the state $|G\rangle$ the cloud transitions to state $|R\rangle$ and thereby blocks the photon passage, whereas in the state $|R\rangle$ it is transparent to the photon. The superposition can be controlled by an atom located in a neighboring potential, which interacts with the cloud via long-range dipole forces such that depending on whether it is in state $|g\rangle$ or $|r\rangle$, the cloud is put in the state $|G\rangle$ and $|R\rangle$. Preparing the control atom in an intial superposition state then produces the required superposition of the Rydberg cloud acting as the quantum obstacle.

\section{PHYSICAL INTERPRETATION AND CONCLUSIONS}

Counterfactual communication belies our intuitive expectation that for Bob to send information to Alice, a physical particle must be exchanged between them. The counterfactual generation of entanglement, of the kind presented here, accentuates this puzzle.

One point worth noting about cryptography in the counterfactual scenario is the following: It might appear that because no physical travel happens in the open channel during counterfactual instances, thus the information has ultimate security. But this is not so [17, since Eve's intervention can physicalize the particle. Thus security must be judged on the basis of a traditional analysis of observed visibilities, photon counts, details of an eavesdropper's attack, etc. All the same, the issue of usefulness of counterfactual communication for cryptography and long-distance communication is an area worth investigating.

From a quantum foundations perspective, counterfactual quantum communication appears to support an ontic interpretation of the wave function that is independent of any particular ontological framework. During a 
$D_{1}$ detection, there was no physical travel of the particle, in that, a physical travel would have resulted in absorption. Yet Bob's choice does influence Alice's observation, given that he communicates information. In order to maintain the philosophical viewpoint that Bob's action is related to her observation not by a remote influence but through a continuous movement of some cause or information in physical space [20], we are led to ascribe some sort of reality to the wave function (the vacuum state, cf. 20]) that propagates from Alice to Bob, and back to her. This conclusion does not require any ontological framework [28], but instead requires only operational considerations about communication (cf. 29]).

Here we summarize how our scheme for counterfactual distribution of entanglement (and for counterfactual quantum information transfer built on top of it) is distinct from the counterfactual schemes proposed in Refs. [21, 22]. The scheme in [21] is one for deterministic counterfactual quantum state transfer, requiring no classical communication and that in 22 first deterministically and counterfactually distributes state-dependent entanglement, which can be used for deterministic quantum state transfer with a 1-bit classical communication (Quantum information transfer of lower fidelity is possible without the classical assistance). In contrast to these two schemes, our scheme is probabilistic. It bears a similarity to the scheme of 22 in that it distributes statedependent entanglement, which can subequently be used for deterministic quantum state transfer using one bit of classical communication. The probabilistic nature of our scheme means (as discussed in Section V) that on average, the minimum number of photons required for the counterfactual generation of state-dependent entanglement is $C_{q}^{\text {min }}=8$ particles, with beam splitter reflectivity $R=\frac{1}{2}$. Further, the minimum average classical communication cost for counterfactual quantum state transfer is $1+C^{\text {min }} \approx 4.85$ bits, with reflectivity $R=\sqrt{2}-1 \approx 0.414$. From an experimental perspective, of significance is that the schemes of [21, 22] are based on CQZE, which requires chaining the actions of a number of beam split- ters. whereas our scheme is probabilistic and uses the experimentally simple Michelsen interferometer set-up.

Our method of generalizing the counterfactual distribution of bipartite entanglement to multi-partite cat states, essentially by making the quantum obstacle in the N09 system to act jointly on multiple particles, can also be applied to CQZE systems. To see this suppose that $N$ copies of the $L$-cycle system in Eq. (1) being vertically stacked, with the obstacle being applied jointly to all $N$ stack layers. Then Eq. (1) becomes:

$$
\begin{aligned}
\mid \text { block }\rangle|0\rangle^{\otimes N} & \left.\longrightarrow \cos ^{L N} \theta \mid \text { block }\right\rangle|0\rangle^{\otimes N} \\
\mid \text { pass }\rangle|0\rangle^{\otimes N} & \longrightarrow \mid \text { pass }\rangle(\cos (L \theta)|0\rangle+\sin (L \theta)|1\rangle)^{\otimes N}
\end{aligned}
$$

As before let $\theta=\pi / 2 L$ and $L \rightarrow \infty$. Then, Bob's obstacle in the state $\alpha \mid$ pass $\rangle+\beta \mid$ block $\rangle$ leads to:

$$
\begin{aligned}
(\alpha \mid \text { pass }\rangle & +\beta \mid \text { block }\rangle)|0\rangle^{\otimes N} \\
\longrightarrow & \left.\alpha \mid \text { pass }\rangle|1\rangle^{\otimes N}+\beta \mid \text { block }\right\rangle|0\rangle^{\otimes N},
\end{aligned}
$$

an $(N+1)$-particle cat state, which generalizes the bipartite superposition of Eq. 22. By placing the above system in an external $M$-chain of interferometers, so that each of the $N$ layers in the above vertical stack is a CQZE $L$-in- $M$ cyclic interferometer, one can analogously implement a deterministic counterfactual distribution of a $(N+1)$-cat state in place of state $(3)$, that extends the bi-partite scheme of [22].

Finally, we briefly mention some future directions opened up by our work. Our study, which considers the noiseless case, may be extended to the open system situation, which would be relevant for purposes of practical implementation. Multipartite quantum information processing protocols based on conventional methods of entanglement generation can be readily adapted to ones based on the present counterfactually generation entanglement.

\section{ACKNOWLEDGMENTS}

RS acknowledges support from the DST project SR/S2/LOP-02/2012.
[1] R. Jozsa, in Lecture Notes in Computer Science, Vol. 1509, edited by C. P. Williams (Springer, 1998) p. 103.

[2] G. Mitchison and R. Jozsa, "Counterfactual computation," Proc. Roy. Soc. Lond. A457 , 1175-1194 (2001).

[3] A. C. Elitzur and L. Vaidman, "Interaction-free measurements," Found. of Phys. 23, 987 (1993).

[4] P. G. Kwiat, H. Weinfurter, T. Herzog, A. Zeilinger, and M. A. Kasevich, "Interaction-free measurement," Phys. Rev. Lett. 74, 4763-4766 (1995).

[5] G.-C. Guo and B.-S. Shi, "Quantum cryptography based on interaction-free measurement," Phys. Lett. A 256, 109 (1999).

[6] O. Hosten, M. T. Rakher, J. T. Barreiro, N. A. Peters, and P. G. Kwiat, "Counterfactual quantum computation through quantum interrogation," Nature 439, 949-952 (2006).

[7] L. Vaidman, "Impossibility of the counterfactual computation for all possible outcomes," Phys. Rev. Lett. 98, 160403 (2007)

[8] T.-G. Noh, "Counterfactual quantum cryptography," Phys. Rev. Lett. 103, 230501 (2009)

[9] Y. Sun and Q.-Y. Wen, "Counterfactual quantum key distribution with high efficiency," Phys. Rev. A 82, 052318 (2010).

[10] S. Zhang, X.-T. Liu, and B. Zhang, "Direct counterfactual quantum communication with high efficiency," ArXiv:1410.2769.

[11] Z.-Q. Yin, H.-W. Li, W. Chen, Z.-F. Han, and G.- 
C. Guo, "Security of counterfactual quantum cryptography," Phys. Rev. A 82, 042335 (2010).

[12] S. Zhang, J. Wang, C.-J. Tang, and Q. Zhang, "Security proof of counterfactual quantum cryptography against general intercept-resend attacks and its vulnerability," Chin. Phys. B 21, 060303 (2012).

[13] S. Zhang, J. Wang, and C. J. Tang, "Counterfactual attack on counterfactual quantum key distribution," Europhys. Lett. 98, 30012 (2012).

[14] H. Salih, Z.-H. Li, M. Al-Amri, and M. S. Zubairy, "Protocol for direct counterfactual quantum communication," Phys. Rev. Lett. 110, 170502 (2013).

[15] A. Shenoy H., R. Srikanth, and T. Srinivas, "Semicounterfactual cryptography," Europhys. Lett. 103, 60008 (2013).

[16] J.-L. Zhang, F.-Z. Guo, F. Gao, B. Liu, and Q.-Y. Wen, "Private database queries based on counterfactual quantum key distribution," Phys. Rev. A 88, 022334 (2013).

[17] A. Shenoy H., R. Srikanth, and T. Srinivas, "Counterfactual quantum certificate authorization," Phys. Rev. A 89, 052307 (2014).

[18] H. Salih, "Tripartite counterfactual quantum cryptography," Phys. Rev. A 90, 012333 (2014).

[19] Z.-Q. Yin, H.-W. Li, Y. Yao, C.-M. Zhang, S. W., W. Chen, G.-C. Guo, and Z.-F. Han, "Counterfactual quantum cryptography based on weak coherent states," Phys. Rev. A 86, 022313 (2012); G. Brida, A. Cavanna, I. P. Degiovanni, M. Genovese, and P. Traina, "Experimental realization of counterfactual quantum cryptography," Laser Phys. Lett. 9, 247 (2012); Y. Liu, L. Ju, X.-L. Liang, S.-B. Tang, G.-L. S. Tu, L. Zhou, C.-Z. Peng, K. Chen, T.-Y. Chen, Z.-B. Chen, and J.-W. Pan, "Experimental demonstration of counterfactual quantum communication," Phys. Rev. Lett. 109, 030501 (2012).

[20] L. Vaidman, "Comment on 'Protocol for direct counter- factual quantum communication'," Phys. Rev. Lett. 112, 208901 (2014) H. Salih, Z.-Hong Li, M. Al-Amri, and M. S. Zubairy, "Salih et al. reply:," Phys. Rev. Lett. 112, 208902 (2014), N. Gisin, "Optical communication without photons," Phys. Rev. A 88, 030301 (2013); L. Vaidman, "Counterfactaulity of counterfactual communication," ArXiv:1410.2723.

[21] H. Salih, "Protocol for counterfactually transporting an unknown qubit," ArXiv:1404.2200.

[22] Q. Guo, L.-Y. Cheng, L. Chen, H.-F. Wang, and S. Zhang, "Counterfactual quantum-information transfer without transmitting any physical particles," Sci. Rep. 5, 8416 (2014).

[23] M. Nielsen and I. Chuang, Quantum computation and quantum information (Cambridge, 2000).

[24] D. Wineland, "Superposition, entanglement, and raising schrodingers cat," Rev. Mod. Phys. 85, 1103-1114 (2013), nobel lecture.

[25] S. Gerlich et al., Nature Comm. 2, 263 (2011).

[26] P. G. Kwiat, A. G. White, J. R. Mitchell, O. Nairz, G. Weihs, H. Weinfurter, and A. Zeilinger, "Highefficiency quantum interrogation measurements via the quantum zeno effect," Phys. Rev. Lett. 83, 4725-4728 (1999)

[27] M. Müller, I. Lesanovsky, H. Weimer, Büchler, H. P., and P. Zoller, "Mesoscopic rydberg gate based on electromagnetically induced transparency," Phys. Rev. Lett. 102, 170502 (2009); J. C. Garcia-Escartin and P. ChamorroPosada, "Counterfactual rydberg gate for photons," Phys. Rev. A 85, 032309 (2012).

[28] N. Harrigan and R. W. Spekkens, "Einstein, incompleteness, and the epistemic view of quantum states," Found. Phys. 40, 125 (2010).

[29] A. Shenoy H. and R. Srikanth, "The wave-function is real but nonphysical: A view from counterfactual quantum cryptography," (2013), arXiv:1311.7127. 